\title{
Ray-traced troposphere slant delays for precise point positioning
}

\author{
Thomas Hobiger $^{1}$, Ryuichi Ichikawa ${ }^{1}$, Tomoji Takasu ${ }^{2}$, Yasuhiro Koyama ${ }^{1}$, and Tetsuro Kondo ${ }^{1}$ \\ ${ }^{1}$ Kashima Space Research Center, National Institute of Information and Communications Technology, \\ 893-1 Hirai, Kashima, Ibaraki 314-8501, Japan \\ ${ }^{2}$ Tokyo University of Marine Science and Technology, 4-5-7 Konan, Minato-ku, Tokyo 108-8477, Japan
}

(Received February 14, 2008; Revised March 24, 2008; Accepted March 28, 2008; Online published May 3, 2008)

\begin{abstract}
Precise satellite orbits and clock information for global navigation satellite systems (GNSS) allow zerodifference position solutions, also known as precise point positioning (PPP) to be calculated. In recent years numerical weather models (NWM) have undergone an improvement of spatial and temporal resolution. This makes them not only useful for the computation of mapping functions but also allows slant troposphere delays from ray-tracing to be obtained. For this study, such ray-traced troposphere corrections have been applied to code and phase observations of 13 sites from the International GNSS Service (IGS) receiver network, which are located inside the boundaries of the Japanese Meteorological Agency (JMA) meso-scale weather model, covering a period of 4 months. The results from this approach are presented together with a comparison to standard PPP processing results. Moreover the advantages and caveats of the introduction of ray-traced slant delays for precise point positioning are discussed.
\end{abstract}

Key words: GPS, troposphere, ray-tracing, PPP.

\section{Introduction}

Precise point positioning (PPP) is a simple and straightforward method which allows the estimation of site coordinates from un-differenced code and phase measurements. Troposphere errors are one of the main contributors to the total error budget and are usually taken into account by parametrization of atmosphere delays as unknown parameters which are estimated together with the other target parameters. The introduction of new mapping functions (NMF and IMF, Niell (1996, 2000, 2001); GMF and VMF, Boehm et al. (2006a, b)) in the recent years significantly improved the results of space geodetic techniques and made it possible to realize sub-cm accurate PPP applications. Although modern mapping functions are derived from numerical weather models, the information from such meteorological data-sets is reduced to a few time- and location-dependent coefficients which relate slanted troposphere quantities to equivalent zenith measures. Moreover, the spatial variations of the troposphere above each station have to be estimated in the analysis process in the form of gradient parameters (e.g. Chen and Herring (1997) or Meindl et al. (2004)). As numerical weather models of regional size have undergone an improvement in terms of accuracy and precision, it has become feasible to utilize ray-traced troposphere slant delays directly for the geodetic analysis of space geodetic techniques. The introduction of these troposphere total slant delays will make the estimation of gradient parameters obsolete and thus reduce the number of unknown parameters.

Copy right(c) The Society of Geomagnetism and Earth, Planetary and Space Sciences (SGEPSS); The Seismological Society of Japan; The Volcanological Society of Japan; The Geodetic Society of Japan; The Japanese Society for Planetary Sciences; TERRAPUB.

\section{Numerical Weather Models and Ray-tracing}

The Japanese Meteorological Agency (JMA) provides a variety of weather models ranging from global models to fine-mesh models which cover an area of only a few tens of kilometers. The meso-scale 4D-Var model (i.e. Mesoscale Analysis Data (MANAL)) from JMA (JMA, 2002; Ishikawa, 2001) with its horizontal resolution of about $10 \mathrm{~km}$ was found to have the best trade-off between gridspacing and area size. This model covers large parts of Eastern Asia, including Japan and its Southern islands, Korea, Taiwan and Eastern China (Fig. 1 shows the model boundaries of the JMA meso-scale model). Moreover the 3-h time-resolution of the data-sets makes the appliance of this model for positioning applications feasible. A couple of programs, called Kashima Ray-tracing Tools (KARAT, Hobiger et al., 2007) re-grid and interpolate the numerical weather models and prepare binary files for follow-on processing. KARAT also handles orbit files and computes the observing geometry for any given RINEX (Gurtner, 2000) file, under the condition that the receiver is located within the boundaries of the meso-scale weather model. In the final step, the ray-tracing itself is carried out for each observation, and the calculated troposphere total slant delays are subtracted from code- and phase-measurements before modified RINEX files are output.

\section{Analysis Strategy}

Although numerical weather models have become highly accurate and ray-traced delays are differing from GNSS derived quantities by only a few millimeters on average, single ray differences ray can reach several centimeters. This is also reflected by a larger RMS of the differences between GNSS and NWM delays (e.g., Haase et al., 2001). Thus it is necessary to account for numerical weather model 


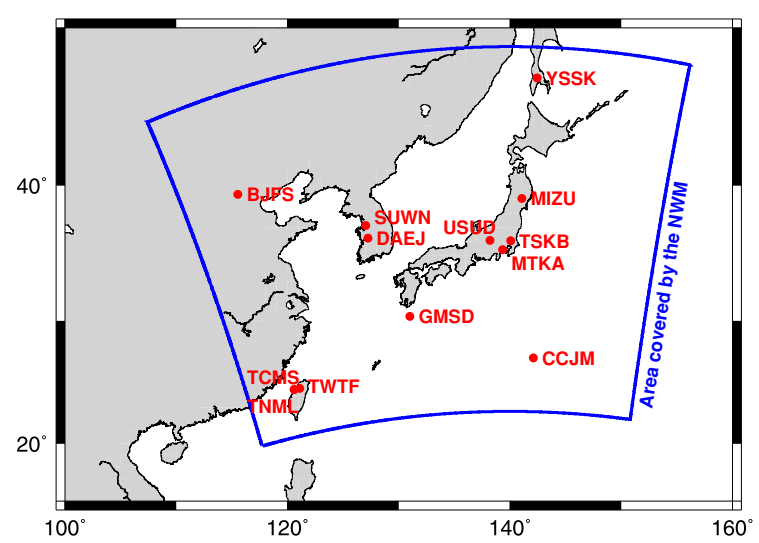

Fig. 1. GNSS receivers of the IGS network which are located within the boundaries of the JMA meso-scale weather model and are included in the daily IGS SINEX solution files, considered for this study.

imperfectness within the parameter estimation process, if ray-traced data is introduced. Since all asymmetric effects, which look like gradients or higher order spatial atmosphere distortions, are expected to be considered properly by the weather models, a residual troposphere zenith delay $\Delta d_{z}(t)$ should be sufficient to compensate for NWM errors $\Delta d(\varepsilon, t)$. Such a correction can be estimated together with the other unknowns within the parameter estimation process via a basic mapping function

$$
\Delta d(\varepsilon, t)=\frac{\Delta d_{z}(t)}{\sin \varepsilon},
$$

which is only dependent on the elevation angle $\varepsilon$. The timevariation of the residual zenith delay can be modeled either by piece-wise linear functions (as in a least-squares adjustment) or taken into account by a random-walk process in a Kalman filter estimation run. Compared to traditional analysis strategies, an approach using ray-traced data does not need a-priori hydrostatic delay information within all steps of PPP processing, and thus does not depend on surface meteorological data. Moreover, it is mandatory that a-priori hydrostatic delays are turned off during code-clock synchronization, ambiguity resolution, and final computation of the receiver position. One draw-back of the introduction of ray-traced slant delays is clearly given by the fact that it is not possible to obtain total or wet troposphere zenith delays as can be done with traditional estimation strategies. Total zenith delays can be reconstructed only when the estimated residual delays $\Delta d_{z}(t)$ are added to ray-traced troposphere zenith delays, which have to be computed together.

Many modern analysis software packages enable the user to compute accurate PPP solutions with a variety of mapping functions that usually include the $1 / \sin \varepsilon$ model, as denoted in Eq. (1). Setting the a-priori hydrostatic delays to zero in the concerned modules mentioned above is not always possible or can be only realized after modifications of the source-code or the creation of artificial meteorological files.

\subsection{GPSTOOLS}

A MATLAB-based set of programs, compiled under the name GPSTOOLS (Takasu and Kasai, 2005), allows to be estimated geodetic target parameters using convenient graphical user interfaces. Small modifications of the main modules were made in order to treat the ray-traced observations properly. Because GPSTOOLS uses a Kalman filter for the estimation of the unknown parameters, the stochastic model for the residual troposphere delays $\Delta d_{z}(t)$ can be set to a random-walk process, which allows the estimates to follow closely to the highly varying (wet) troposphere. Parameter estimation within GPSTOOLS is done by a forward/backward Kalman filter using user-defined update intervals and stochastic models. GPSTOOLS utilizes IGS final orbit and clock products and estimates PPP solutions in the IGb00 reference frame, after smoothing the forward/backward Kalman filter solutions. GPSTOOLS takes slightly more computation time than comparable analysis packages which are coded closer to machine level, but provides many useful tools for data-screening and display of results.

\subsection{Receiver network}

In order to test the impact of ray-traced troposphere slant delays on PPP estimates RINEX data from selected IGS sites have been obtained for a 4-month period starting on November 5, 2006, and lasting until February 28, 2007. Limitations on an extension of the analysis time-span were determined by the availability of numerical weather model data at the time of preparing this paper. Data before November 5, 2006 was not considered for this study since results degrade when relative phase center variation (PCV) models are applied. In total, 13 IGS stations have been selected, which are inside the spatial domain of the JMA meso-scale model and are considered in the routine daily IGS analysis procedure. Figure 1 depicts the locations of the selected stations, distributed over East Asia. All receivers continuously tracked the GNSS satellites, with the exception that one or the other receiver lost data for single days. As some of the receivers are located very close to each other, it is expected that any systematic errors introduced by numerical weather models will be reflected by similar differences between the estimated position and the IGS solution. Thus, RINEX files were downloaded, screened for irregularities and repaired if necessary using GPSTK (Harris and Mach, 2007) in order to guarantee that KARAT will not encounter any troubles due to data-gaps or RINEX format inconsistencies. Based on these data, a second set of RINEX files has been generated for which the troposphere slant delays from ray-tracing calculations have been removed.

\subsection{Parametrization}

Analysis runs were carried out with the original (hereafter called "standard solution") and ray-traced data-set ("KARAT + residual troposphere solution"). In order to demonstrate how KARAT performs when the troposphere residual is not estimated a "KARAT-only solution" has been computed for reference. A cut-off elevation angle of 10 degrees was chosen for all runs in order to ensure that troposphere effects can be clearly separated from clock and height parameters. For the standard solution, the a-priori hydrostatic zenith delays were computed from the Saastamoinen (1972) model based on standard atmosphere values. The global mapping function (GMF, Boehm et al., 2006a) and linear gradient estimation (Chen and Herring, 1997) have been chosen for the troposphere modeling. Elevation- 
Table 1. Repeatabilities and RMS of the residuals for KARAT-only (denoted by "K-O"), KARAT + residual troposphere ("K+R") and standard solution ("S") are listed for each receiver. Receivers marked by a star do not have calibrated absolute phase center variation models.

\begin{tabular}{|c|c|c|c|c|c|c|c|c|c|c|c|c|}
\hline \multirow[t]{2}{*}{ Site } & \multicolumn{3}{|c|}{ Repeat. East (mm) } & \multicolumn{3}{|c|}{ Repeat. North (mm) } & \multicolumn{3}{|c|}{ Repeat. Up (mm) } & \multicolumn{3}{|c|}{ RMS res. (mm) } \\
\hline & $\mathrm{K}-\mathrm{O}$ & $\mathrm{K}+\mathrm{R}$ & $\mathrm{S}$ & $\mathrm{K}-\mathrm{O}$ & $\mathrm{K}+\mathrm{R}$ & $\mathrm{S}$ & $\mathrm{K}-\mathrm{O}$ & $\mathrm{K}+\mathrm{R}$ & $\mathrm{S}$ & $\mathrm{K}-\mathrm{O}$ & $\mathrm{K}+\mathrm{R}$ & $\mathrm{S}$ \\
\hline BJFS & 4.3 & 3.8 & 3.9 & 2.3 & 2.2 & 2.3 & 17.4 & 4.8 & 5.1 & 8.5 & 7.1 & 7.2 \\
\hline CCJM* & 6.2 & 4.0 & 4.0 & 4.0 & 3.0 & 2.4 & 32.7 & 6.6 & 6.6 & 14.3 & 9.6 & 9.2 \\
\hline DAEJ* $^{*}$ & 4.2 & 3.6 & 4.0 & 2.6 & 2.3 & 2.4 & 16.5 & 4.9 & 4.7 & 8.6 & 7.0 & 7.0 \\
\hline GMSD & 5.5 & 3.9 & 4.3 & 2.9 & 2.6 & 2.5 & 20.9 & 7.0 & 8.1 & 10.1 & 8.4 & 8.5 \\
\hline MIZU & 4.2 & 3.9 & 4.2 & 4.2 & 3.8 & 4.3 & 15.3 & 7.7 & 7.7 & 9.4 & 7.7 & 7.8 \\
\hline MTKA & 5.1 & 3.8 & 3.9 & 3.2 & 2.8 & 3.1 & 20.5 & 5.7 & 6.1 & 9.4 & 7.6 & 7.8 \\
\hline SUWN & 4.8 & 4.1 & 4.4 & 2.4 & 2.1 & 2.2 & 16.8 & 5.4 & 5.1 & 8.8 & 7.2 & 7.2 \\
\hline TCMS & 5.9 & 4.1 & 3.9 & 3.8 & 3.1 & 2.6 & 49.1 & 5.9 & 6.1 & 12.9 & 9.1 & 8.6 \\
\hline TNML & 6.3 & 4.4 & 4.3 & 4.0 & 3.3 & 2.7 & 51.0 & 5.8 & 5.8 & 13.0 & 8.7 & 8.2 \\
\hline TSKB & 4.5 & 3.6 & 3.6 & 2.6 & 2.3 & 2.6 & 18.8 & 4.7 & 4.7 & 9.6 & 7.3 & 7.4 \\
\hline TWTF* & 6.0 & 4.8 & 4.7 & 3.7 & 3.0 & 2.7 & 45.1 & 5.8 & 5.8 & 12.6 & 8.7 & 8.3 \\
\hline USUD & 4.6 & 4.3 & 4.5 & 3.3 & 3.0 & 3.2 & 13.7 & 7.0 & 8.2 & 7.8 & 6.7 & 6.9 \\
\hline YSSK ${ }^{*}$ & 4.5 & 4.2 & 4.6 & 2.5 & 2.3 & 2.7 & 13.0 & 5.0 & 5.2 & 7.6 & 6.4 & 6.5 \\
\hline Average & 5.1 & 4.0 & 4.2 & 3.2 & 2.7 & 2.7 & 25.4 & 5.9 & 6.1 & 10.5 & 7.9 & 7.8 \\
\hline
\end{tabular}

dependent weighting has been carried out by varying the measurement noise proportional to $\sim c_{0} / \sin \varepsilon$ where $c_{0}$ has been set $6 \mathrm{~mm}$, which is the standard parametrization of GPSTOOLS. The Kalman-filter estimation interval was set to $300 \mathrm{~s}$, without overlapping data from consecutive days. The second processing, using the ray-traced RINEX files, used identical parametrization except that the a-priori troposphere delays were set to zero and a simple mapping function (Eq. (1)) was selected. In a final stage, the obtained station coordinates were de-trended and station position repeatabilities were computed for all sites.

\section{Results}

Table 1 shows the East-, North- and Up-repeatabilities of all three solutions. It can be seen that the analysis of KARAT data without troposphere residual estimation gives quite comparable horizontal positioning performance, but yields worse stability of the height component. This can be explained by the fact that all deficits of the numerical
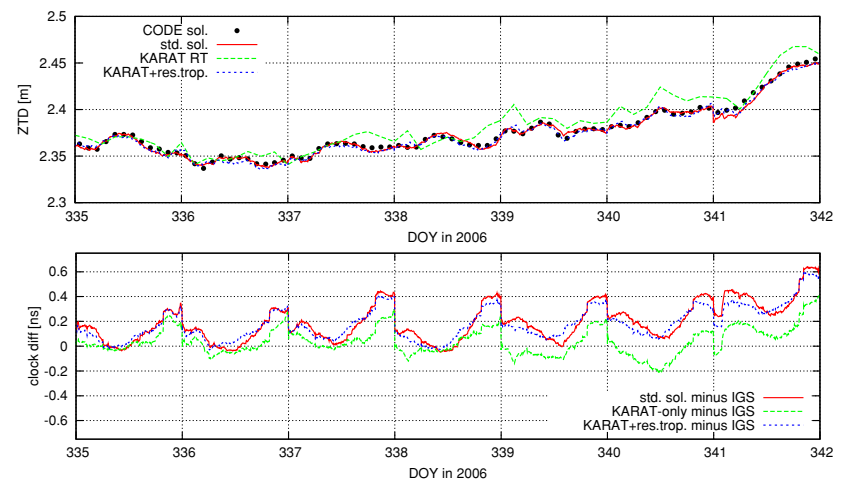

Fig. 2. Nuisance parameters for station TSKB from December 1, 2006 OUT until December 8, 2006 0UT. Upper plot: Zenith total delays (ZTDs) from the standard solution (continuous line), from ray-tracing (dashed line) and ray-tracing + estimated residuals (dotted line). Additionally the solution from the Center for Orbit Determination in Europe (CODE) has been added for reference (dots). Lower plot: Differences of the receiver clock estimates with respect to the IGS combined solution. weather model translate (mainly) into the height component. If KARAT data are used together with troposphere residuals estimation, the station height repeatabilities are improved for nearly all sites compared to the standard solution. GMSD and USUD particularly benefit from the ray-traced data set by reducing the height scattering by more than $1 \mathrm{~mm}$. Stations which are not located inside the Japanese territories, for which the JMA model is expected to be more accurate, as well as stations with non-calibrated absolute PCV models seem to have slightly larger height repeatabilities. On average, KARAT data reduce the height repeatability by about $3 \%$, whereas the horizontal measures are only slightly improved. The RMS of the residuals from the KARAT + residual troposphere and the standard solution are nearly identical, whereas the KARAT-only solution has again larger values. A test with KARAT data, troposphere residual estimation and gradient parametrization showed that the ray-traced observations are free of any gradient-like structures as the estimated values are at least 20 times smaller than the gradients obtained from the standard solution. Moreover, the formal errors of the obtained gradient parameters exceed the estimated values by at least one order of magnitude when the KARAT data-set is used. It is anticipated that linear gradient estimation, as carried out in the standard solution, also absorbs other asymmetric error sources, such as un-modeled phase center variations or multi-path effects. In addition, it has been studied how the use of the ray-traced data impacts the estimation of the nuisance parameters. Figure 2 shows zenith total delays and clock estimates at station TSKB between December 1, 2006 0UT and December 8, 2006 0UT as an example and relates them to corresponding IGS products (note: since IGS ZTD combination has been discontinued since Nov. 5, 2006 the solutions of the Center for Orbit Determination in Europe (CODE) have been used). The ZTDs computed from KARAT were overlain to the GPSTOOLS estimates in order to demonstrate that the numerical weather models are able to cover more than $99 \%$ of the total troposphere delay, but lack of temporal resolution and millimeter accuracy. Thus it is still mandatory to estimate the resid- 
ual troposphere (see Section 2) in order to avoid biases of the target parameters caused by NWM imperfectness. If the estimated troposphere residuals are added to the raytraced ZTDs, the solution is nearly identical to the standard solution, for which the troposphere has been parametrized by dry and wet constituents. The clock estimates from the KARAT + residual troposphere and the standard solution agree well with each other. Moreover it is interesting to see that the KARAT-only solution agrees best with the IGS clock estimates. This is thought to be caused by the fact that, although the station height might be wrong by 1 or $2 \mathrm{~cm}$, the clock can be separated properly since no mapping function (which is only accurate to a certain level) is needed.

\section{Discussion and Outlook}

Numerical weather models have been improved in terms of accuracy and spatial resolution which makes it possible to utilize them for the correction of troposphere delays of precise point positioning. However, since these models are still not capable of providing total troposphere delays with millimeter accuracy it is necessary to estimate these residual delays by a simple mapping function together with the other unknowns. By applying this strategy, the raytraced data performs slightly better than the standard PPP analysis, which is based on modern mapping functions and linear gradient estimation.

As the current analysis tools are designed to estimate wet-troposphere delays with a magnitude of several tens of centimeters it will be necessary to revise the stochastic model of the Kalman filter in order to take into account the fact that the residual troposphere delays are only a few centimeters at maximum. Thus, it is necessary to revise measurement- and process-noise components together with the filter initialization parameters, to obtain the best performance from the ray-traced data.

Beside the simple mapping function $(\sim 1 / \sin (\varepsilon))$ applied here, tests have been carried out using NMF and GMF for the reconstruction of the residual troposphere zenith delays using the KARAT data. All three mapping functions yielded the same performance, which can be explained by the fact that the estimated residual ZTDs are in the millimeter to centimeter range and therefore mapping function errors at lower elevation do not have so much impact as they do on the estimation of wet zenith delays in standard processing of GNSS data. Additionally, a 1 month data-set from October 2006 has been analyzed showing that the relative phase center models, which have to be used for all data before Nov. 5, 2006, do not permit an improvement of the height repeatability. Some of the sites benefitted from the KARAT data, but others showed a degradation of the height repeatabilities. Thus, accurate absolute receiver phase-center variation models (e.g. Schmid et al., 2007) are necessary to take full advantage of the ray-traced slant delay information. Moreover, it is anticipated that multi-path mitigation strategies will further improve the performance of the KARAT data and reduce the RMS of the residuals.

Since numerical weather models will be continuously improved in terms of spatial and temporal resolution, the performance of the proposed analysis strategy will also increase in the future. Additionally, the usage of ray-traced observations does not need any big modifications of existing analysis software packages and allows a reduction of the number of unknowns within the adjustment process. For applications with lower accuracy requirements and kinematic positioning tasks, such ray-traced data can already be applied without any estimation of residual troposphere delays, yielding centimeter-range accurate positioning solutions.

Acknowledgments. We are very grateful to the Japanese Society for the Promotion (project P06603) for supporting our research. The International GNSS Service is acknowledged for providing observational data and products. We want to thank Dr. Böhm and one anonymous reviewer for the valuable comments that led to significant improvements of our paper.

\section{References}

Boehm, J., A. Niell, P. Tregoning, and H. Schuh, Global Mapping Function (GMF): A new empirical mapping function based on numerical weather model data, Geophys. Res. Lett., 33, L07304, doi:10. 1029/2005GL025546, 2006a.

Boehm, J., B. Werl, and H. Schuh, Troposphere mapping functions for GPS and very long baseline interferometry from European Centre for Medium-Range Weather Forecasts operational analysis data, J. Geophys. Res., 111, B02406, doi:10.1029/2005JB003629, 2006b.

Chen, G. and T. A. Herring, Effects of atmospheric azimuthal asymmetry on the analysis of space geodetic data, J. Geophys. Res., 102(B9), 20,489-20,502, doi:10.1029/97JB01739, 1997.

Gurtner, W., RINEX: The Receiver-Independent Exchange Format, GPS World, 5(7), 48-52, 2000.

Haase, J. S., H. Vedel, M. Ge, and E. Calais, GPS zenith tropospheric delay (ZTD) variability in the Mediterranean, Phys. Chem. Earth, Solid Earth Geodes., 26(6-8), 439-443, 2001.

Harris, R. Benjamin and R. G. Mach, GPSTk-An Open Source GPS Toolkit, GPS Solutions, 11(2), doi:10.1007/s10291-006-0043-7, 2007.

Hobiger, T., R. Ichikawa, Y. Koyama, and T. Kondo, Kashima Ray-Tracing Service (KARATS) — Fast ray-tracing through numerical weather models for real-time positioning applications, NICT IVS Technical Development Center News, 82, 16-19, 2007.

Ishikawa, Y., Development of a mesoscale 4-dimensional variational data assimilation (4D-Var) system at JMA, Proceedings of the 81st Annual Meeting of the AMS: Precipitation Extremes: Prediction, Impacts and Responses, P2.45, 2001.

JMA, Outline of the operational numerical weather prediction at the Japanese Meteorological Agency, 158 pp, 2002.

Meindl, M., S. Schaer, U. Hugentobler, and G. Beutler, Tropospheric Gradient Estimation at CODE: Results from Global Solutions, J. Meteorol. Soc. Jpn., 82, 331-338, doi:10.2151/jmsj.2004.331, 2004.

Niell, A. E., Global mapping functions for the atmosphere delay at radio wavelengths, J. Geophys. Res., 101(B2), 3227-3246, 1996.

Niell, A. E., Improved atmospheric mapping functions for VLBI and GPS, Earth Planets Space, 52, 699-702, 2000.

Niell, A. E., Preliminary evaluation of atmospheric mapping functions based on numerical weather models, Phys. Chem. Earth, 26, 475-480, 2001.

Saastamoinen, J., Atmospheric correction for the troposphere and stratosphere in radio ranging of satellites, The Use of Artificial Satellites for Geodesy, Geophys. Monogr. AGU, 15, 247-251, 1972.

Schmid, R., P. Steigenberger, G. Gendt, M. Ge, and M. Rothacher, Generation of a consistent absolute phase-center correction model for GPS receiver and satellite antennas, J. Geodes., 81(12),781-798, doi:10. 1007/s00190-007-0148-y, 2007.

Takasu, T. and S. Kasai, Evaluation of GPS Precise Point Positioning (PPP) Accuracy, IEIC Technical Report, 105(208), 40-45, 2005.

T. Hobiger (e-mail: hobiger@nict.go.jp), R. Ichikawa, T. Takasu, Y. Koyama, and T. Kondo 